Marquette University

e-Publications@Marquette

Mathematics, Statistics and Computer Science Mathematics, Statistics and Computer Science, Faculty Research and Publications

$10-2017$

\title{
The Double-edged Sword: A Mixed Methods Study of the Interplay between Bipolar Disorder and Technology Use
}

\author{
Mark Matthews \\ Cornell University \\ Elizabeth Murnane \\ Cornell University \\ Jaime Snyder \\ University of Washington \\ Shion Guha \\ Marquette University, shion.guha@marquette.edu \\ Pamara Chang \\ University of Cincinnati
}

See next page for additional authors

Follow this and additional works at: https://epublications.marquette.edu/mscs_fac

Part of the Computer Sciences Commons, Mathematics Commons, and the Statistics and Probability Commons

\section{Recommended Citation}

Matthews, Mark; Murnane, Elizabeth; Snyder, Jaime; Guha, Shion; Chang, Pamara; Doherty, Gavin; and Gay, Geri K., "The Double-edged Sword: A Mixed Methods Study of the Interplay between Bipolar Disorder and Technology Use" (2017). Mathematics, Statistics and Computer Science Faculty Research and Publications. 561.

https://epublications.marquette.edu/mscs_fac/561 


\section{Authors}

Mark Matthews, Elizabeth Murnane, Jaime Snyder, Shion Guha, Pamara Chang, Gavin Doherty, and Geri K. Gay

This article is available at e-Publications@Marquette: https://epublications.marquette.edu/mscs_fac/561 


\section{Marquette University \\ e-Publications@Marquette}

\section{Mathematics, Statistics, and Computer Science Faculty Research and Publications/College of Arts and Sciences}

This paper is NOT THE PUBLISHED VERSION; but the author's final, peer-reviewed manuscript. The published version may be accessed by following the link in the citation below.

Computers in Human Behavior, Vol. 75 (October, 2017): 288-300. DOI. This article is $(\subset$ Elsevier (Cell Press) and permission has been granted for this version to appear in $\underline{\mathrm{e}-}$ Publications@Marquette. Elsevier (Cell Press) does not grant permission for this article to be further copied/distributed or hosted elsewhere without the express permission from Elsevier (Cell Press).

\section{The Double-Edged Sword: A Mixed Methods Study of the Interplay Between Bipolar Disorder and Technology Use}

Mark Matthews

Information Science, Cornell University, Ithaca, NY

Elizabeth Murnane

Information Science, Cornell University, Ithaca, NY

Jaime Snyder

Information Science, University of Washington, Seattle, WA

Shion Guha

Department of Mathematics, Statistics and Computer Science, Marquette University, Milwaukee, WI

Pamara Chang

Department of Communication, University of Cincinnati, Cincinnati, $\mathrm{OH}$

Gavin Doherty

Computer Science, Trinity College Dublin, Ireland

Geri Gay

Information Science, Cornell University, Ithaca, NY 


\section{Abstract}

Human behavior is increasingly reflected or acted out through technology. This is of particular salience when it comes to changes in behavior associated with serious mental illnesses including schizophrenia and bipolar disorder. Early detection is crucial for these conditions but presently very challenging to achieve. Potentially, characteristics of these conditions' traits and symptoms, at both idiosyncratic and collective levels, may be detectable through technology use patterns. In bipolar disorder specifically, initial evidence associates changes in mood with changes in technology-mediated communication patterns. However much less is known about how people with bipolar disorder use technology more generally in their lives, how they view their technology use in relation to their illness, and, perhaps most crucially, the causal relationship (if any exists) between their technology use and their disease. To address these uncertainties, we conducted a survey of people with bipolar disorder $(N=84)$. Our results indicate that technology use varies markedly with changes in mood and that technology use broadly may have potential as an early warning signal of mood episodes. We also find that technology for many of these participants is a double-edged sword: acting as both a culprit that can trigger or exacerbate symptoms as well as a support mechanism for recovery. These findings have implications for the design of both early warning systems and technologymediated interventions.

\section{Keywords}

Serious mental illness; Bipolar disorder; Technology use; Self-tracking; Relapse detection; mHealth

\section{Abbreviations \\ BD Bipolar Disorder \\ 1. Introduction}

The evolution and rapid dissemination of mobile and sensor technology creates unprecedented opportunities to study human behavior in an extremely granular, unobtrusive, and continuous manner. This increased mediation of human activity via technology has particular potential for individuals with bipolar disorder (BD) who display distinct changes in behavioral and cognitive processes at different phases of their illness. Bipolar disorder affects approximately $2.4 \%$ of the population worldwide and is recognized as one of the most debilitating illnesses - responsible for more disability-adjusted life years than all forms of cancer (Merikangas et al., 2011). BD is characterized by extreme changes in energy and mood between the highs of a manic episode and the lows of a depressive episode (Merikangas et al., 2011). These changes are often accompanied by significant changes in sleep, physical activity, social behaviors, and goal-directed activities. However, while common classes of warning signs as well as symptoms of relapse for bipolar disorder are well identified, little is known about their online and technologically-mediated manifestations. 
Recent studies in mental health and technology have provided initial evidence that changes in mental health can be detected by changes in communication patterns (Frost, Doryab, FaurholtJepsen, Kessing, \& Bardram, 2013) including online textual content (De Choudhury, Gamon, Counts, \& Horvitz, 2013, p. 2). There has been an increasing focus on developing usercentered technologies to support the management of bipolar disorder (e.g (Faurholt-Jepsen et al., 2015, Matthews et al., 2016).,), and symptom detection and predict is a desirable capability of such tools. Yet we still know relatively little about broader patterns of technology use from the perspective of people diagnosed with bipolar disorder.

The study of technology use by people with bipolar disorder is important because it may lead to advances in the early detection of mood episodes. For example, a continuous monitor of technology use may help replace burdensome and unreliable self-report questionnaire that are still the dominant method to protect against relapse. This effort may also result in a better understanding of the manifestation of bipolar disorder, at least online, which may in turn fuel the development of protective and supportive digital tools. Finally, understanding how people with this condition use technology both broadly and to self-manage their condition will inform how best to develop digital interventions that are user-centered. Thus, knowing more about the use of technology by people with bipolar disorder could lead to identifying novel methods to prevent relapse, identifying novel and improving existing digital treatments.

In this present study, we seek to provide a rich picture, from the perspective of people who have bipolar disorder, about how technology fits into their lives. We have three explicit goals:

- to characterize how people with bipolar disorder use technology in their lives

- to investigate whether people with bipolar disorder perceive manifestations of manic or depressive episodes in their technology-mediated behavior

- to identify both positive and negative impacts of technology use on participants' condition

To address these questions, we conducted an online survey of individuals $(\mathrm{N}=84)$ who have bipolar disorder. We find that digital behavior resembles in many ways real world behavior during manic and depressive episodes. Participants report general increased activity during elevated mood states, and more passive or less frequent digital interactions during depressed mood states. For the first time, we also provide specific examples and personal testimonies of the ways technology use changes across states and various risk and supportive aspects of this very use (See Fig. 1).

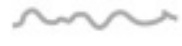

Normal

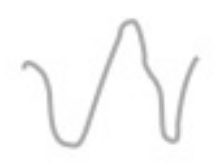

Bipolar I

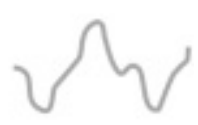

Bipolar II

Fig. 1. A comparison of mood variation among mood in the general population and the two subtypes of bipolar disorder, BD-I and BD-II. 


\section{Background}

\subsection{Bipolar disorder characteristics}

Bipolar disorder affects a person's cognition, mood, and energy levels. Individuals with bipolar disorder are frequently severely disabled, and their rate of completed suicide is least 15 times that of the general population (Harris \& Barraclough, 1997). There is no cure for bipolar disorder. After onset, it lasts a lifetime and without treatment can progressively become more debilitating. It is therefore not surprising that this prevalent, life-threatening, and lifelong illness (Murray \& Lopez, 1996) is associated with poor functional and clinical outcomes (Judd et al., 2003), high suicide rates (Baldessarini \& Tondo, 2003), and huge societal costs (Pari et al., 2014).

The Diagnostic and Statistical Manual of Mental Disorders (DSM-5) (American Psychiatric, 2013), the most recent version of the psychiatric manual for diagnosing mental illnesses, categorizes bipolar disorder as a mood disorder defined by a cycle of manias, where the individual experiences high self-esteem, irritability, and sleeplessness as well as severe depressions. There are several sub-types of this disease, most prominent of which are BD-I and BD-II.

Bipolar I Disorder (BD-1) is the most severe form of BD. About $0.6 \%$ of the population will experience the condition at some point in their life (Merikangas et al., 2011). The main difference from other subtypes is the degree to which life is impaired and the possible presence of psychosis (e.g., delusions, which are fixed false beliefs or hallucinations, such as hearing voices when no one is speaking). To receive a diagnosis of BD-I, individuals must experience at least one manic episode, including 7 days or longer of "abnormally and persistently elevated, expansive, or irritable mood and abnormally and persistently increased goal-directed activity or energy" (American Psychiatric, 2013). This is also coupled with marked changes in behavior most often characterized by a global increase in physical and mental activity, as well as a decreased need for sleep.

Bipolar II (BD-II) is less severe and slightly less common than BD-I - 0.4\% (Merikangas et al., 2011). The key difference is that individuals diagnosed with BD-II do not experience manic episodes but rather less severe hypomanic periods, a milder state that shares the symptoms of BD-I but with the absence of psychosis and less elevated and abnormal mood and energy. Significant numbers of people outside of these categories experience some symptoms, with a lifetime prevalence of 1.4\% for sub-threshold BD and 2.4\% for Bipolar Spectrum Disorder (Merikangas et al., 2011).

\subsection{Comorbid conditions}

It is important to note that people with bipolar disorder are almost never dealing with just their bipolar disorder. Most people with BD (70\%) suffer from 3 or more disorders over the course of their lifetime (Merikangas et al., 2007). Most people with bipolar disorder suffer from some form of anxiety such as panic attacks and social phobia (Merikangas et al., 2007). Substance abuse is also common in bipolar disorder; one study found that $60 \%$ of individuals sampled 
had some history of substance abuse (Merikangas et al., 2011). Further, people with BD tend to experience more physical health complaints, including an increased risk of cardiovascular disease, diabetes, and pulmonary conditions (Merikangas et al., 2011). In addition, as the number of experienced relapses increases, so does the risk for cardiac disease, substance abuse, and suicide. In short, living with several comorbid psychological and health conditions complicates the day-to-day management of health and may have an impact on the range of technologies individuals use to stay on top of their treatment and more broadly.

\section{Related work}

Existing literature regarding the relationship between technology use and mental health has typically focused on the use of technology for mental health management and most often from a clinical perspective. Initial work has provided some evidence that real-world and technologymediated behavior can provide signals about mental health status and symptoms, and the beneficial or problematic associations between technology and wellbeing (e.g., social support or internet addiction). Overall, the range and substance of this work provides evidence that technology can both have positive and negative effects on mental health.

\subsection{Technology for mental health monitoring}

Wearable technology and mental health monitoring technologies have shown some success in helping to track and manage bipolar disorder. One prototype system combined self-monitoring with wearable sensors to track behavioral manifestations of BD symptoms (Blum \& Magill, 2010). Researchers have also designed a shirt that integrates fabric electrodes and sensors that acquire health information including electrocardiogram, respirogram, and body posture in order to detect depressive, mixed, and hypomanic mood states (Valenza et al., 2014).

Attempts at passive mobile sensing have found high correlations between smartphone use patterns and BD patients' self-reported mood, sleep, and irritability (Alvarez-Lozano et al., 2014). Frost et al. developed a smartphone app to provide better disease insights by collecting data relevant to the behavioral trends of bipolar disorder (Frost et al., 2013). Based on data collected over six months, they found that patient mood correlated with physical activity, levels of stress, sleep and phone use. Another study used phone call duration, speech analysis and movement data from smartphones to identify manic and depressive states (Grünerbl et al., 2015). From twelve weeks of data with ten patients, their system recognized bipolar states and the transitions between those states (assessed using the Hamilton Rating Scale for Depression and Young Mania Rating Scale) with 76\% accuracy.

\subsection{Online manifestations of mental health}

Research to-date has mainly focused on how online social systems in particular may reflect mental health. Some work has found that social media use may predict symptoms for a range of personality and mood disorders. One recent study administered the Millon Clinical Multiaxial Inventory (MCMI-III; (Millon, Millon, Davis, \& Grossman, 2009)) for psychological assessment along with questions about technology-related attitudes and anxieties in the context of 
Facebook and found that more Facebook use, increased displays of impression management on Facebook, and more Facebook friends were significantly predictive of clinical symptoms of personality disorders (e.g. schizophrenia) and mood disorders (e.g. bipolar-mania) (Rosen, Whaling, Rab, Carrier, \& Cheever, 2013) - though the participants $(N=1143)$ had no confirmation or clinical diagnosis for these conditions.

Regarding bipolar disorder specifically, a cross-sectional study of 30 euthymic (e.g., stable) BD patients with matched controls examined whether the online social behavior of individuals with bipolar disorder mirrors their social behavior in the real world (Martini et al., 2013). Using a questionnaire to assess social behavior online, the researchers found that the effects of BD on social functioning did extend to electronic media. Specifically, BD individuals self-reported significantly lower numbers of both Facebook friends as well as lifetime-estimated numbers of offline contacts.

\subsection{Beneficial and problematic technology use}

Generally, evidence suggests that the use of communication and information technology can benefit mental well-being if that mediated interaction is supportive. Online communication that builds relationships or fosters a sense of community has been shown to have a positive effect (Magsamen-Conrad \& Greene, 2014), and research finds that using the internet to communicate with close connections can diminish symptoms of depression (Bessiere, Kiesler, Kraut, \& Boneva, 2008). However, use that is excessive or driven primarily by a desire to avoid social isolation is associated with loneliness, unhappiness, and increased depression (Kross et al., 2013, Muusses et al., 2014). Such problematic use can produce psychological distress (Caplan, 2003) as well as stress and sleep disturbances (Thomée, Härenstam, \& Hagberg. 2011).

Similarly, while technology use may provide valuable signals to detect patterns of mental health, it may also agitate or even trigger symptoms of disordered states. For example, exposure to the bright light emanated by devices has been shown to cause sleep disruptions and disorders (Wood, Rea, Plitnick, \& Figueiro, 2013), which is problematic given that sleepwake stability is a key factor for emotion regulation for BD (Harvey, 2008). In addition, the always-on mentality and endless-streaming nature associated with technology and online media in particular can produce and exacerbate feelings of preoccupation, compulsion, and anxiety, which in turn increase stress and depressed mood (Harwood, Dooley, Scott, \& Joiner, 2014).

Finally, some prior work has suggested associations between bipolar disorder and internet addiction (IA) or problematic internet use (PIU) (Kardefelt-Winther, 2014). In a recent study conducted on Korean adolescents, researchers surveyed 795 middle school and high school students on questions regarding and pulled from the Internet Addiction Proneness Scale, frequencies of depression, suicidal ideations, and PIU (Park, Hong, Park, Ha, \& Yoo, 2013). Results showed that there is a complex relationship and association between PIU, depressive symptoms, bipolar symptoms, and suicidal ideations. Specifically, the presence of problematic Internet use was significantly associated with suicidal ideations and depression, and there was a significant association between problematic Internet use and bipolar disorder. 


\subsection{Novel contributions}

Existing research thus provides initial evidence that, at least for small samples, changes in tech-mediated behavior can be associated with changes in offline behavior, mood, and mental health status. However, studies have investigated technology-mediated behavior and patterns of use in a relatively narrow band (e.g., Facebook or text messaging), whereas technology use by individuals with $\mathrm{BD}$ is likely more diverse.

Further, studies on technology and BD generally focus on use specifically during manic and depressive episodes. It is important to also study patterns and habits of use when individuals are balanced as well as explore whether there are meaningful aspects about how individuals manage their health using technology or put protective strategies in place to maintain balance.

In this paper, the aim is to address these issues by contributing insights into how broad forms of technology are used by individuals with bipolar disorder, including during times of affective balance and during mood episodes.

\section{Materials and methods}

\subsection{Survey instrument}

To explore relationships between technology and BD from the perspective of individuals with the disorder, we developed a questionnaire with 49 items including a mix of close-ended, Likert-scale, and open-ended questions. The instrument has three sections: technology use, relationship between technology use and bipolar disorder, and demographics. We discuss our choice of survey items in the following sections. The complete survey can be found in the Appendix.

\subsubsection{Technology use}

First, we aim to explore technology ownership and use amongst the population of individuals with bipolar disorder who already use technology. Very little is known about the ownership and use of technology which is relevant to understanding both the feasibility of developing technology-based interventions and whether technology use itself might serve as a valid measure of wellbeing in this population. Hence, we incorporate questions from the ECAR study of University students and technology about ownership and use frequency of devices and software (Dahlstrom, Walker, \& Dziuban, 2013), along with a set of adapted questions in order to assess individuals' "enthusiasm" for technology including the adoption of new technology.

As mentioned, some prior work has suggested associations between bipolar disorder and Internet addiction or problematic Internet use. In order to assess whether addiction to technology was a factor in our sample's reported use of technology, we included a reduced set of measures (in the interests of completion time) from the Internet Addiction Test (Young, 1998). 


\subsubsection{Self-reported signals and indicators of behavior changes}

Known changes in behavior (e.g., increased communication, racing thoughts, and poor concentration during a mood episode) might be detectable from a person's patterns of technology use. To assess this, we included custom questions related to participants' perceptions of how their technology use changes with aspects of their BD and whether they have identified ways in which their BD symptoms manifest through particular usage patterns. Beyond whether technology use might provide signals of the onset of bipolar episodes, we are also interested in whether a causal relationship exists between technology use and manifestations of $\mathrm{BD}$. With this in mind, we incorporate questions about personal technologymediated BD triggers. Uncovering such risk factors could support the development of improved treatment strategies including appropriate technology-based approaches.

\subsection{Data collection}

Our survey instrument was distributed by the International Bipolar Foundation to its mailing list, and the survey was also posted on WeSearchTogether.org, a website that helps researchers find participants for studies on mood disorders. Data were collected between September 23rd, 2013 and October 8th, 2014.

Recruitment was directed at anyone with a confirmed diagnosis of bipolar disorder, although diagnosis was self-reported. Anonymous completion of the survey was possible. However, as incentive for participating, individuals had the option to enter their email address into a drawing to win an iPad, which would of course diminish anonymity. The Institutional Review Boards of the authors' academic institutions approved all procedures.

A total of 112 participants from 8 countries completed our survey. Of these, 84 were fully complete, valid responses. 71 of the validated respondents were women, and 71 were from the United States. Respondents are not evenly distributed across all age and BD categories. Rather, they tend to be less than 35 years old, mostly have Type I and Type II bipolar disorder, and have experienced at least 10 episodes through their life. For a more complete description of our participants, please see Table 1.

Table 1. Summary of age of first episode, bipolar type, and number of lifetime episode.

Age of 1st episode

Type of bipolar disorder

Episodes

BD-I BD-II BD not otherwise specified Cyclothymia Other

$\begin{array}{lllllll}\text { less than } 18 & 16 & 20 & 2 & 1 & 3 & 42 \\ 18-24 & 11 & 9 & 4 & 0 & 1 & 25 \\ 25-34 & 4 & 4 & 3 & 0 & 1 & 12 \\ 35-44 & 2 & 2 & 0 & 0 & 0 & 4 \\ 45-54 & 1 & 0 & 0 & 0 & 0 & 1 \\ \text { Total } & 34 & 35 & 9 & 1 & 5 & 84\end{array}$




\subsection{Analysis}

The purpose of this study is to gain a better understanding of the interplay between bipolar disorder and technology use. We used a mixed-methods approach to analyze survey responses. Specifically, we combined (a) a qualitative analysis of self-reported patterns and perceptions of technology use in order to obtain insights about individual practices with (b) descriptive and inferential statistical analyses of several characteristics of participants' technology use in order explore a preliminary model of relationships among these factors and the illness.

\subsubsection{Qualitative analysis}

Open-ended questions were used to explore the effect of technology on individuals' everyday lives and on aspects of their bipolar disorder. The responses to these open-ended and freetext questions were analyzed in several stages.

First, the same questions and corresponding responses were assigned to two of the authors, such that each response was read by both coders. Each author worked iteratively and independently to develop a list of themes and seek out patterns for each question using open coding (Lofland \& Lofland, 1995). The two authors then met and discussed the themes and patterns that each had observed, noting both similarities and differences among responses to different questions.

Based on the discussion, the authors enhanced their original list of codes, themes, and patterns. This list of codes became a basis for the second stage of analysis, which was to code the rest of the responses to all the open-ended questions. In this stage, the two authors both read every response to each question. Once the coding was complete, the two authors met again to have further discussions about the codes. Disagreements were discussed and resolved between the two authors. The purpose of the discussion process was to serve as another iteration in developing and redefining our understanding of the codes and themes in the data and the relationships among them.

\subsubsection{Quantitative modeling of technology use}

A number of closed-ended questions were included in an effort to model the relationship among respondents' symptoms and various aspects of their technology use. To do so, we constructed 3 ordinal regression models.

We defined the following Independent Variables about participants:

- TechUseFrequency: A matrix representing the average frequency of use for each type of technology or device use as reported by participants.

- TechUseModality: A count variable representing each unique technology type or device a participant reports using. 
- AddictionScaleScore: A continuous variable representing the outcome of a participant's responses to the series of internet addiction questions.

- TechEnthusiasm: A continuous variable representing the outcome of a participant's responses to the questions assessing enthusiasm and adoption of technology.

- PersonalTriggers: A binary variable representing a participant's awareness of any personal triggers of a manic or depressive episode that relate to the use of technology

We also incorporated the following Control Variables into our models:

- Gender: A binary variable (male or female). We offered other choices to participants but all of them responded as either male or female.

- Age: A categorical variable with 9 levels adapted from the US Census ranging from "under 18 years" to "85 years or over"

- Marital Status: A categorical variable with 8 levels adapted from the US Census ranging from "single, never married" to "in relationship, not living together"

- Bipolar Disorder Type: A categorical variable with 4 levels as defined in the DSM.

- No. of Episodes: A count variable describing the cumulative number of episodes experienced by a participant.

- Age of First Episode: A categorical variable with 8 levels identical to "Age" defined above which describes the age in which participants had their first bipolar disorder episode.

Finally, 3 questions were included to assess whether participants believe their technology use varies prior to or during episodes or remains constant, using custom 5-point Likert type scale ranging from "Strongly Disagree" to "Strongly Agree". As the goal of these models is to explore the potential for correlating bipolar disorder episodes with self-perceived technology use behaviors, we therefore tested for significant predictors of the following Dependent Variables, which represent this self-reported degree to which participants' technology use varies (or does not vary) with bipolar symptoms:

- TechUsePriorEpisode: whether participants believe their technology use broadly changes prior to a mood episode

- TechUseDuringEpisode: whether participants believe their technology use broadly changes during a mood episode

- TechUseConstantEpisode: whether participants believe their technology use broadly remains constant 


\section{Results}

We organize our findings around our three main questions:

1. How do participants make use of technology, including ownership and usage frequency? (Section 5.1)?

2. How do symptoms of bipolar disorder manifest through patterns of technology use? (Section $\underline{5.2}$ )?

3. What is the nature of the relationship between technology use and bipolar disorder? (Section $\underline{5.3}$ )?

\subsection{Technology use}

Most participants (71.4\%) consider themselves enthusiastic about and comfortable with electronics and digital devices, and only $14.2 \%$ report that they find technology frustrating. While $15 \%$ of respondents report never using a smartphone, all but one of the remaining respondents use a smartphone every day. Smartphones, laptop computers and tablets are the most commonly owned and frequently used devices, while fewer respondents use desktop computers. Table 2 presents reported ownership and usage levels for various electronic devices.

Table 2. Device ownership and use levels.

\section{Technology}

Smartphone (e.g., iPhone, Android phone, Blackberry, etc.)

Desktop Computer Laptop/Netbook Computer

Tablet Computer (e.g., iPad)
Ownership Use daily or Use once a week Use once a 2-3/week or 2-3/month month or less $85 \%$

$71 \%$ $85 \%$ $80 \%$
$100 \%$

$35 \%$

$75 \%$

$100 \%$ $0 \%$ $0 \%$
Focusing on frequency of software use, most participants (85\%) use email, text messaging, and Facebook a few or many times a day (averaging 8, 22, and 24 times per day, respectively) while Twitter is used less (only $30 \%$ of participants use it at least once per day). See Table 3 for more details. Open-ended responses also suggest that text messaging is more popular than instant messaging for respondents since they associate the former with contacting closer connections and it is easily accessible from phones. Facebook and playing computer games have a binary split between participants who use them every day and non-users. 
Table 3. Software use levels.

$\begin{array}{llll}\text { Technology } & \begin{array}{c}\text { Use once a } \\ \text { day or more }\end{array} & \begin{array}{c}\text { Use a few times a } \\ \text { week/month }\end{array} & \begin{array}{c}\text { Use less than once a } \\ \text { month/Never use }\end{array} \\ \text { Text Messaging } & 85 \% & 15 \% & 0 \% \\ \text { Email } & 85 \% & 15 \% & 0 \% \\ \text { Twitter } & 30 \% & 30 \% & 40 \% \\ \text { Computer Games } & 30 \% & 0 \% & 70 \% \\ \text { Facebook } & 85 \% & 0 \% & 15 \% \\ \text { Instant Messaging (Gchat, } & 40 \% & 30 \% & 30 \% \\ \text { Facebook chat, AIM, etc.) } & & & \end{array}$

For respondents who report using these types of software a few times a day or many times per day, Table 4 presents specifics about the average amount of content or time they spend on that software. In particular, we find that text messaging and Facebook are most frequently used for communication but also that the time spent playing computer games is considerable.

Table 4. Daily use for respondents who report using software a few or many times per day.

\section{Technology}

Text Messaging

Email

Twitter

Computer Games

Facebook

Instant Messaging (Gchat, Facebook chat, AIM, etc.)

\section{Average Use Per Day}

Send 50 messages, Receive 35

messages

Send 15 emails, Receive 25 emails

Post 3 tweets

Play for $5 \mathrm{~h}$

Check 40 times

Send 25 messages

We next look more carefully into how patterns of technology use during such periods may serve as a signal of manic and depressive symptoms.

\subsection{Symptomatic technology use}

\subsubsection{Perceptions of prodromal technology use}

$41 \%$ of respondents report changes in their technology use that serve as personal warning signs of oncoming manic or depressive episodes. Looking at responses to our adapted internet addiction questions, we find that excessive technology use is not uncommon for respondents, especially at night and just prior to or during an episode. When asked to describe specific examples of technology-based warning signs, one respondent reports associating oncoming mania with an increased sense of anxiety stemming from technology, for instance, from 
notification sounds of incoming emails and text messages. Generally, respondents explain that their amount and frequency of use begins to escalate, especially late at night, as mania approaches:

"It's a build up. Sometimes it is a struggle to get off especially at night. Then the cycle of not sleeping/technology use at night starts." (P43)

Conversely, oncoming depression is signaled by an increasing detachment from technology for many respondents:

"If I am barely using computer it means I am probably doing little else which is a sign I am entering a phase dominated by depressive symptoms." (P5)

As another respondent puts it:

"If my social media interactions lessen or increase it can be signs of episodes. If I ignore technology and communications with others via text etc., I am more than likely heading to a depressive episode". (P3)

\subsubsection{Variations in technology use during BD episodes}

Qualitative data provides additional granular detail about the ways technology use varies for some individuals during episodes. 59\% of respondents agree or strongly agree that their technology use habits change during bipolar episodes, $17 \%$ disagree, $4 \%$ strongly disagree, and $20 \%$ are not sure. Asked about specific manifestations of mood shifts, respondents provide many examples (summarized and categorized in Table 5) of the ways in which the amount and timing of use as well as the types of technology being used can all exhibit distinct qualities during manic and depressive periods as compared to balanced periods.

Table 5. Variations in technology usage that are characteristic of mania and depression.

\section{Indicator}

Computer-mediated communication (e.g., email, messages - to the point it may be phone, text messages, Facebook messages, tweets, blog posts)

Social networking (e.g., $\quad$ Checking social news feeds repeatedly Facebook, Twitter)

Web searching and browsing

\section{Manic Manifestations} construed as spam — or writing an excess of online content

Obsession with performing web searches or managing and rapidly switching among multiple browser windows and tabs
Depressive Manifestations Avoiding email or dodging phone calls and texts

Avoidance of social media

Non-use or "idle" use (e.g., reading websites) 
Indicator

Streaming media (e.g.,

Netflix, Hulu)

E-commerce

Gambling and gaming

Digital calendars

Typing and audio
Manic Manifestations

"Binge watching"

Compulsively online shopping

Obsessively gambling or playing games on computers or phones for hours - especially "calming" games social games, high-action games, or multiple (e.g., solitaire) games at once

Excessively booking activities

Diminished use

Faster, more careless use (e.g., more typos Slower use or more garbled speech)

Technology-mediated risky Increased visitation of dating or pornography None reported behavior sites, sending $X$-rated photos, using more inappropriate and aggressive language in written content, or more risk-oriented web searches (e.g., to find tattoo parlors or research exuberant vacations)

Use timing and frequency Late night use: excessively checking phone Diminished use notifications; or paranoid checking partners' overall emails, social media accounts, or cell phone logs)

First, the responses illustrate that mania and depression are marked by extremes of technology overuse and underuse. During manic periods, respondents "envelop" themselves in technology, for instance becoming consumed with performing web searches and rapidly switching among multiple browser windows and tabs (i.e. context switching). Hyperactivity during respondents' manic periods also manifests through the excessive checking of phone notifications or booking of activities in digital calendars. Respondents also associate mania with faster, more garbled speech patterns, which technology-mediated communications analogously reflect:

"I'm told I post more to various websites and am hyperverbal even with typing." (P4)

Noted types of technology overuse include obsessively gambling online, playing games on computers or phones, and compulsively shopping on the internet. Expenditure spikes and spending sprees are known to often occur offline during bipolar episodes. Individuals in our study similarly report increases in online spending and shopping during manic phases. According to one respondent:

"EBay is a real danger to me. I love to 'snipe' to win. In the last 3 months I bought 9 Gucci watches .... but I am back under control again now. Now repairing the damage to 
my finances! And I am a chartered accountant by profession, trained at Anderson and PWC!" (P54)

Online expenditure may also better satisfy a desire for instantaneous satisfaction and reward, which is thought to increase when an individual is in a hypomanic state (Nusslock, Abramson, Harmon-Jones, Alloy, \& Coan, 2009).

Just as online shopping leads to reward-seeking gratification, spontaneous relationships and the outcomes from these interactions may be similarly gratifying. Respondents in our study report increased risk-seeking social behaviors such as visiting more dating or pornography sites, sending X-rated photos, and using more inappropriate and aggressive language in written content. Web searches similarly become more risk-oriented. For example, they are used to find tattoo parlors or research exuberant vacations:

"During a manic episode, I find myself using much less safety online. I often give out my cell phone number or email address to people that I would not normally give it out to, I engage in sexual behaviors that I would never consider while not manic, I tend to contact people from my past more often and flirt, I send X-rated messages and photos." (P18)

In contrast, respondents identify a substantial drop in technology use prior and during depressive periods. When individuals do use technology when depressed, they describe using it in more "passive" and "zombie" like ways, for instance by "idly" reading content, "mindlessly" watching TV shows, or only "checking on" others' social media sites rather than initiating interactions. Respondents contrast this sort of use during depression a way to escape from negative emotions compared to the more "proactive" interactions characteristic of mania:

"During Mania: Marked increase in online activity. Numerous tabs open on my browser on a variety of topics. During Depression: Marked increase in TV viewing. (Do not want to move from the couch, don't even want to get up and get the remote to change the channel)." (P33)

"Sometimes I withdraw completely from proactive interaction. I may still "observe" on Facebook or groups, but not post. Usually before/during depression." (P43)

"manic: lots of active use and communication and online interactions. depressive: lots of passive use: reading news and articles" (P50)

Thus not only do respondents' technology-mediated activities appear to reflect their BD symptoms, but manic and depressive states often present themselves in antithetical ways. Distinct changes in the use of social technology during manic or depressive periods emerge as particularly salient. Increased sociality is a hallmark of mania, while social isolation is known correlate of depression (Bruce \& Hoff, 1994). Respondents report repetitively (re)reading and sending texts, Facebook messages, and emails - to the point it may be construed as spam during mania as well as writing an excess of blog posts, tweets, or other online content. Respondents also mention checking Facebook and other social news feeds over and over; 
and paranoia and anxiety associated with mania sometimes lead them to check their partners' emails, social media accounts, or cell phone logs.

On the other hand, respondents describe "withdrawing from the world" — both offline and online during depression. They explain this social detachment and isolation manifests in avoidance of social media and email, dodged phone calls and texts, and a complete lack of interest in the same technology-mediated social activities that were obsessively sought during times of mania:

"When I'm in a depressive state I don't want any interaction with anyone. When I'm in a manic state I constantly talk and my technology use increases." (P45)

In summary, almost three-quarters of respondents described ways that their BD manifests through changes in their technology use prior to and during episodes. Although specific manifestations vary by individual, broadly speaking, mania and depression are marked by extreme increases and decreases in technology use. Levels of use escalate as mania approaches, especially for social technologies (in line with the connection between sociality and mania) and drop markedly before and during depressive periods. During those periods, respondents describe withdrawing from the world both offline and online, leading to avoidance of the same technology-mediated activities sought during times of mania. When individuals do use technology when depressed, they describe using it in more mindless and passive ways, mostly as an escape to distract them from negative emotions.

\subsection{Negative impacts of technology use on BD}

Having just uncovered ways in which patterns of technology use can signal oncoming or current states of mood disturbance for our respondents, we next look into whether certain patterns of technology use may not only reflect but also impact mood. $41 \%$ of respondents report being aware of personal triggers of manic or depressive episodes that are related to the use of technology. When asked about specific examples, respondents describe issues related to social distress, exposure to certain types of content, and excessive and overstimulating latenight use.

\subsubsection{Social comparison}

First, just as offline social isolation is a known precursor and symptom of depression, individuals describe that feeling socially excluded online is similarly disruptive to emotional balance. In particular, social media is noted as a culprit causing a sense of jealousy, missed opportunity, and being ignored or left out, which can all contribute to feelings of loneliness and hopeless - and ultimately lead to or amplify depression:

"[My personal triggers of episodes are] seeing friends on Facebook and all they have accomplished and how happy they look." (P27)

"Getting on Facebook and feeling jealous as others have such rich lives. (I am no longer on fb for this reason). I closed my account 2 years ago." (P21) 


\subsubsection{Negative content}

Respondents report that exposure to pornography or posts with sexual innuendo can trigger mania. Frustrating experiences with technology such as software crashes, encountered bugs, unexpected performance, or cumbersome interactions are also noted as agitating. Most prominently, participants report that spending too much time with technology is overstimulating and can fuel a spiral into mania.

Regarding depression, respondents explain that negative content such as inflammatory or offensive posts and disturbing news stories can produce anxiety and paranoia and in turn, trigger or reinforce their depressive symptoms:

"I generally avoid too much news programming by avoiding TV, because the state of the world can overwhelm any positivity I can muster." (P74)

\subsubsection{Impact of technology use on sleep}

In our data, participants identify late-night technology use as a disruptor to sleep, which is especially troublesome given that sleep instability is a trigger of circadian imbalance and in turn, mood disruption for bipolar individuals (Harvey, 2008). 45\% of respondents report always or often staying online longer than they intend or saying "just a few more minutes" when online, including at bedtime. Nearly three-quarters of respondents keep their smartphone bedside their bed at night, and most respondents (67\%) report losing sleep due to late-night use at least occasionally.

Responses to open-ended questions about the effect of technology on sleep reflect similar findings, with participants expressing that they stay up longer than intended due to technology, have difficulty falling asleep even once they put the technology down, and get fewer hours of sleep because of it all. This is exemplified in the following comment from a participant that identifies interlocking issues of addiction, oversharing, and sleep disruption:

"I often feel anxious about not being connected, and I spend too much time online, especially on Facebook. I used to use Twitter and LiveJournal every day, but I've managed to cut back on those drastically, and I don't overshare nearly as much. But I will definitely forego sleep just so I can check my email and Facebook." (P46)

In a few cases, respondents use technology to occupy themselves when unable to sleep anyway:

“Doesn't affect my sleep, it gives me something to do when I can't sleep." (P16)

"I definitely go to technology before most other avenues when I have trouble sleeping" (P49).

However, participants also suggest that such insomnia may be a symptom of a disrupted sleep routine that could in part be corrected by eliminating pre-bed technology use: 
"One step I took to curb my reliance on technology was to go on a 40-day Facebook cleanse. That distraction being gone has helped me refocus on finding a healthier sleep solution." (P49)

\subsection{Positive aspects of technology use}

Though we have presented a number of negative impacts technology use can have on BD, participants also identify aspects of technology that are beneficial and help to diminish symptoms. Here we briefly overview the positive aspects of technology most discussed by respondents: access to information, self-tracking assistance, social support, and encouraging content.

Prior research on patient self-care has established that information-seeking is a key practice for a number of health conditions (Lambert \& Loiselle, 2007). Responses indicate that this is the case for BD as well: the internet enables participants to research mental health information, gain knowledge about their bipolar disorder, and develop personal coping strategies.

"Research, research, research! Knowledge is empowering - and helps me accept and understand my illness." (P8)

$73 \%$ of participants feel that technology-mediated tracking has helped them improve their health. In describing their use of technology for health management, respondents note a number of items they monitor that are associated with their bipolar condition specifically or how its symptoms personally manifest or get triggered — for example, medication and any side effects, physical symptoms and pain levels, suicidal ideation and self-harm, appetite, libido and intimate relations, and caffeine and alcohol intake. Other self-monitored variables that participants relate to their mood fluctuations include blood pressure, weight, menstrual cycles, water intake, and bathroom visits.

"Without these tools I would not be able to look at how I can improve my mood." (P47)

As presented earlier, social aspects of technology use can sometimes agitate mania or reinforce depression by generating perceptions of anxiety, inferiority, and isolation for participants; yet technology-mediated social interaction can also positively contribute to overall well-being, enhance a sense of connectedness, and help respondents recover from episodes for respondents. They express value in the ability to find social support online - especially by connecting with other individuals who also have bipolar disorder and experience similar problems:

"Social media and online support groups allow me an opportunity to interact with other people, occasionally friends but more importantly other folks that live with the same issues that I do." (P50) 
For our respondents, this ability to connect with fellow people with BD is also important since it helps to counteract perceptions of isolation, stigma, and shame surrounding their disorder, which they tend to encounter when interacting with people without the condition:

"I usually don't discuss it because of the stigma." (P27)

Beyond the interpersonal connectedness inherent to social technology, the content in social media also helps respondents cope with depression. One's own content can be a source of encouragement:

"Looking back at old messages, posts, and pictures helps to remind me of the things that used to make me happy. When I'm depressed sometimes I wonder if I was ever happy at all, and seeing evidence of it really helps." (P62)

Similarly, content posted by others can be uplifting, inspirational, and motivational:

"I use Facebook in a way to help manage my symptoms. I like Facebook pages that post inspirational and motivational quotes. Surprisingly these updates really make a difference for me. Whenever I go on Facebook I can see these words of wisdom and what not and it reminds me what is important in my life, and keeps my attitude positive. However, in a way this could be considered a double-edged sword because I often spend too much time on this task and it can interfere with my other responsibilities." (P12)

\subsection{Elusive causality and feedback loops}

As this last quote suggests, a chicken-and-egg question exists between technology use and bipolar symptoms. Many respondents discuss their uncertainty about whether particular patterns of use generally trigger and fuel bipolar episodes or whether being manic or depressed leads to distinct levels and types of technology use:

"I also start to notice that I lose more and more sleep because I've been up late, usually online ... Is losing sleep triggering episodes or is the episode triggering my staying up late?" (P39)

The combination of technology together with particular energy and mood levels may also push an individual into an episode or exacerbate existing symptoms. For example, heightened energy and mood levels may lead an individual to initiate conversations on a dating site or play games longer than usual - technology-mediated activities we previously identified as related to mania. Engaging in these behaviors might further increase stimulation, impact sleep, and overall spark instability and ultimately fuel a "full blown" episode:

"I use online dating sites and sometimes will do all-nighters talking to a new person. I'm actually not sure, though, if that's a trigger for a manic episode (because of the staying up late), or if it is a symptom of mania. Chicken or egg - I would have to pay more attention next time." (P28) 
Respondents also imply the relationship between technology and symptoms may not simply be one triggering the other but rather a more cyclical process. An example from our data is for an individual to experience stress, lose sleep as a result, increasingly use technology during periods of this insomnia, encounter negative posts online, and consequently experience stress yet again. The dynamics of these feedback loops can depend on an individual's idiosyncratic traits, bipolar condition, and current situation.

In summary, many participants report both positive and negative impacts of their technology use on their condition, as concisely conveyed by one respondent:

"[Technology is a] "double edged sword. Sometimes it's a trigger, sometimes it's a CAUTION sign, sometimes it's a tool, and sometimes it can save your life." (P43)

\subsection{Modeling technology use prior and during episodes}

The primary goal of this study is to contribute an increased understanding of technology use for individuals with bipolar disorder including identifying tech-mediated signals of symptoms. It could be possible to translate those insights into computational approaches for symptom detection and prediction. The findings from participants' qualitative responses suggest that particular patterns of technology use (e.g., rises or drops in the frequency of use or the use of certain types of technology) may serve as signals about a bipolar episode that is approaching or already in progress. While in the future it would be valuable to use actual logged data of tech-mediated behavior in the modeling, for now, we move towards this passive assessment goal by using self-reported data about use behaviors. Specifically, we construct a preliminary model (see Methods section 4.3.2 for details of all variables) to explore the potential for quantitatively predicting such signals of BD symptoms.

Table 6. Results of ordinal regression to predict self-reported variance in technology use during BD episodes.

\begin{tabular}{lllll}
\multicolumn{1}{c}{ Variables } & \multicolumn{1}{c}{ Estimate } & \multicolumn{1}{c}{$\begin{array}{c}\text { Standard } \\
\text { Error }\end{array}$} & \multicolumn{1}{c}{$\begin{array}{c}\text { Effect Size } \\
\left(\boldsymbol{\omega}^{2}\right)\end{array}$} & \multicolumn{1}{c}{ Confidence Intervals } \\
Intercept & $-3.348^{*}$ & 0.459 & 0.214 & $(0.089,0.393)$ \\
TechUseFrequency & $0.52^{* * *}$ & 0.119 & 1.443 & $(1.134,2.181)$ \\
TechUseModality & $0.97^{*}$ & 0.46 & 0.378 & $(0.154,0.664)$ \\
AddictionScaleScore & $0.192^{* * *}$ & 0.057 & 0.715 & $(0.627,0.903)$ \\
TechEnthusiasm & -0.375 & 0.029 & 0.616 & $(0.244,1.388)$ \\
PersonalTriggers & $0.727 *$ & 0.539 & 0.323 & $(0.113,0.978)$ \\
AIC $=124$ & & Deviance $=31.346$ & ${ }^{*} p<0.05{ }^{* *} p<0.01$ \\
& & &
\end{tabular}


Specifically, we obtain a best-fit convergent model for predicting technology use during episodes (TechUseDuringEpisode). Results and fit statistics are presented in Table 6. To summarize, the significant predictors of this model are:

(a) the overall frequency of a participant's technology use (high effect size),

(b) the number of different types or modalities of technology a participant uses in general (moderate effect size),

(c) an increased sense of feeling addicted to technology (high effect size), and

(d) the existence of perceived personal triggers of bipolar episodes stemming from the use of technology (low effect size).

In other words, these results show that the higher a person's baseline level of technology use, the more types of technology a person uses, and the greater a person's general sense of technological attachment, the greater the self-perceived change in technology use patterns during bipolar episodes. Though not significant, we also find a negative association between reported enthusiasm for technology and the change of technology use during an episode. That is, people who report more enthusiasm for using technology and adopting the latest technology report their technology use during episodes does not change much.

Models fail to converge for TechUseConstantEpisode (i.e., patterns of technology use remain constant in response to episodes) and TechUsePriorEpisode (i.e., patterns of technology use change prior to an episode), which is not unexpected given that our qualitative data suggests episodes are associated with distinct changes in technology use but that most of these changes are more apparent during the episode rather than prior to it. Another, more technical reason for failure of these models to converge might be the previously described left-skewed distribution of most of our control variables (see Table 1).

\section{Discussion}

\subsection{Tech ownership \& use}

The potential utility of technology as a means to measure mental health and as a platform for improving health depends in large part on levels of technology use and access in the target population. The penetration of technology including mobile devices among populations with bipolar disorder remains uncertain. One aim of this study was to characterize how people with bipolar disorder use technology in general and specifically their level of mobile phone ownership. The level of mobile phone penetration in populations with serious mental illnesses does appear to be increasing at a similar rate to that of the general population. A recent metaanalysis of mobile phone ownership in populations with psychosis (Firth et al., 2015) revealed an overall ownership rate of $66.4 \%$ across 15 studies $(\mathrm{N}=3227)$, as well as a statistically significant increase in ownership levels since 2007 leading to an ownership rate of $81.4 \%$ $(n=454)$ in the last two years of the study resembling that of the general population which is $90 \%$ (Smith, 2015). In populations with psychosis smartphone ownership is lower, on average only $35 \%$, but higher on average (49\%) across the US, UK and Canada. A study by Ben-Zeev 
suggests that the ownership levels of mobile devices (including cellular phones and smartphones) were higher among those with mood disorders (86\%) than those with schizophrenia/schizoaffective disorder (63\%) (Ben-Zeev, Davis, Kaiser, Krzsos, \& Drake, 2013).

The overall level of technology ownership and use in our sample was very high. Smartphone ownership (our survey did not include a question about cellular phone ownership) is our sample was very high (85\%). This is higher than the level of ownership found in the general population (68\% in the US) in 2015 (Smith, 2015) suggesting that there is a bias in our sample due perhaps to recruiting participants online. This could also be a result of the higher proportion of young respondents in our sample: relatively higher levels of smartphone ownership (of around 68\%) have been observed among younger patients in earlier stages of psychotic disorders (Lal, Dell'Elce, \& Malla, 2015).

\subsection{Symptomatic tech use}

A second aim of this study was to investigate whether people with bipolar disorder perceive manifestations of manic or depressive episodes in their technology-mediated behavior. While the survey does not support generalizations about the ownership rates of technology outside of our limited sample, it does provide evidence that amongst those individuals who own and use technology with a reasonable level of frequency that this use may provide signals that can be used to detect changes in mental state and characterize online manifestations of symptomatic behavior.

\subsubsection{Online manifestations of BD symptoms}

Our findings revealed strong connections between technology use patterns and bipolar episodes. Mania is indicated by hyperactive use behaviors (e.g., checking, posting, searching) -- for example, using multiple devices simultaneously (such as computer and phone, while watching TV), having many browser tabs open, seeking instant gratification through online shopping or gambling/game-playing, and switching among various sources of stimulating content. During periods of severe depression, respondents tend to be more passive (e.g., observing, viewing, withdrawing), withdraw from technology use or even abandon it entirely.

These characteristics associated with mood episodes are good starting points for future work looking at actual data streams from individuals with bipolar disorder. For example, initial analyses might further investigate the relationship between mood and the overall frequency of technology use combined with the timing of use, particularly sustained late night use. Our exploratory model indicated that the frequency of technology use, the types of modalities used, and the level of self-perceived technological addiction were all significant predictors of variations in technology use during manic or depressive phases. This suggests that future analyses might begin by focusing on people with bipolar disorder who use technology a lot and may struggle with addictive elements. 


\subsubsection{Implications of symptomatic tech use}

Prior studies have provided evidence that variations in use of specific software such as Facebook might be indicative of mental health status (De Choudhury et al., 2013, p. 2). Our study provides evidence that it may be possible to proactively identify collective and idiosyncratic manifestations of bipolar disorder through technology use more broadly.

Technology-mediated behavior might serve as a continuous method to monitor mental health that does not place a burden on the individual. This could offer the potential to remove a considerable burden for people with BD to self-monitor their symptoms. The dominant method for tracking symptoms and warning signs in clinical practice with bipolar patients is still paperbased diaries. The shortcomings of this method are well-identified and include: inconsistent completion, unreliable data as a result of retrospective recall (Stone, Shiffman, Schwartz, Broderick, \& Hufford, 2003). Another significant advantage of using digital traces over selfreport is that it can be analyzed retrospectively after a relapse to better understand behavioral manifestations that may serve as warning signs that a person could be vigilant of in the future. From a privacy perspective, our results indicate that content-free analyses of technology use, that is data that only looks at frequency and timing of use, may be sufficient for identifying warning signs for some individuals.

One of the greatest challenges for those experiencing BD is that official diagnosis takes on average 10 years (Suppes et al., 2001). This means that the initiation of therapeutic and/or psycho-pharmaceutical intervention can be delayed for years from the onset of symptoms. Technology-mediated behavior, including analysis of retrospective data stores of for example email patterns or social media use may serve as a method for assisting in arriving at a more rapid and accurate diagnosis. A similar approach may have use in the proactive monitoring among relatives of $\mathrm{BD}$ individuals who are known to have a genetic risk of developing BD.

Finally, while current research indicates that each person's manifestation of bipolar disorder is a unique result of their genetic makeup and surrounding environment, current clinical criteria place patients into accurate but not highly specific categories. Longer term, online technology use may have the potential to provide more nuanced assessments, via what we might call technology-mediated phenotyping, of each individual's manifestation of BD.

\subsubsection{Differences and similarities between tech-mediated symptomatic behavior?}

Our results indicate that some online manifestations of symptoms mirror real-world counterparts. For example, spending sprees have long been associated with manic episodes. Participants in our survey reported spikes in their online purchases on websites like Amazon or Ebay during manic phases. Table 7 identifies reported online manifestations of common symptomatic behaviors. 
Table 7. Showing possible online manifestations of BD phase from our survey.

\section{Symptom}

Impulsive Behavior

Hypersexuality

Racing Thoughts

Decreased Need for Sleep

Goal-directed Activity

Risky Behavior

More Talkative

\section{Online Manifestation}

Amazon, Ebay

Dating sites, sexting, pornography

Multiple tabs, many searches

Up late online, posting on Facebook

Multiple-tabs open, searching, amazon

Gambling, sexting, oversharing

Text messaging, Facebook, Email

In future work, it may be interesting to consider the difference that technology-mediation might have on the follow-on impact of these behaviors. For instance, impulsive behaviors can be more easily taken to an extreme online -- consider online shopping vs. when an individual is required to physically travel from store to store in real life. Also, oversharing online may be more long lasting and challenging to remedy compared to oversharing in face-to-face situations. On the other hand, some behaviors such may actually be less risky online than in situations in real life -- for example consider "sexting" vs. one night stands.

Future research might explore such differences between manifestations of bipolar disorder in real world situations versus when this behavior is mediated by technology. For example, are many of the behaviors that were not relatively common for manic phases such as starting a new business, more easily achieved online? For example, is impulsivity more easily exercised online? A better understanding of these affordances could inform a class of tools that protect individuals with $\mathrm{BD}$ against such online behaviors or diminish their negative impact. One example might be a tool that monitors for spending sprees and either alerts a loved one or provides a straightforward method to reverse these transactions.

\subsection{Effect dynamics between technology use and BD}

The final aim of our study was to identify both positive and negative impacts of technology use on participants' BD. This study has suggested there is a multi-faceted relationship between technology use and individuals' bipolar disorder. This includes the likelihood of bi-directionality between manic or depressive behaviors driving certain types of use or certain types of use triggering or fueling episodes and the positive ways individuals with bipolar use technology to help manage their illness. This is perhaps unsurprising given that technology is not homogenous and mediates a range of human behavior. Participants' reported: (1) technology triggering mood episodes, (2) technology exacerbating episodes, (3) technology use changing as a result of independent symptomatic behavior and (4) technological behavior that improved wellbeing by providing information, social support or increasing motivation.

One area where technology use appears to negatively impact is sleep, which is a key behavioral marker of wellbeing in bipolar disorder. Respondents' interpretation suggests the relationship may in fact be cyclical in nature rather than unidirectional one way or the other -- 
for instance, late night technology use disrupts sleep or stimulating content agitates stability, which subsequently initiates a manic state, which is then further fueled by easy access to the technological culprit. Sleep disturbance and circadian dysregulation are critical pathophysiological factors in bipolar disorder. While light from electronic devices like smartphones and tablets is not thought to shift the circadian phase, it has been shown to have an impact on alertness and delay sleep onset (Wood et al., 2013). This could be particularly negatively impactful in bipolar disorder where sleep and circadian disruption is thought to be a path to relapse (Frank, Swartz, \& Boland, 2007). Similar real world causal factors exist that have a similar impact such as air travel (Miklowitz and Alloy, 1999, Proudfoot et al., 2011). Furthermore, as reported by some participants, the device may not be the initial trigger that disrupts sleep but it may contribute to prolonging or exacerbating sleep disruption.

Prior work provides very initial evidence that individuals with bipolar disorder may be more likely to report feelings of addiction related to their technology use (Park et al., 2013). Our study does not clarify this picture although our exploratory model indicates that higher levels of technology addiction are associated with reported changes in technology use during mood episodes. This suggests that such individuals may be particularly good candidates for technology use as a proxy for mental wellbeing.

Although empirical work on Problematic Internet Use and Internet Addiction have received much focus (Kardefelt-Winther, 2014), argues that much of this work may lack progress and clear results. Therefore, Karedefelt-Winther suggests an alternative model termed Compensatory Internet use to investigate the frequent assumption that individuals go online to escape real life issues or alleviate dysphoric moods that may oftentimes lead to negative outcomes. This alternative model would illuminate the relationship between psychosocial wellbeing and Internet addiction ultimately highlighting how online activities may compensate for psychosocial problems. In the context of this study with individuals with bipolar disorder, this suggested model could help explain why some people continually spend a lot of time online despite experiencing negative outcomes.

Social relationships appear to be a key driver of technology use in both beneficial and detrimental ways. For instance, in hypomanic periods, respondents join numerous dating sites or forums, compulsively check social networking sites, and message and "friend" strangers with whom connections are not maintained after the manic phase ends. Respondents also express that exposure to certain types of such content -- social media in particular -- can induce social anxiety, increase feelings of social isolation, and ultimately trigger depression. They then seek social support through forums, text messaging, and other types of technologymediated social interaction to deal with and recover from depressive phases, since social support is instrumental to coping according to respondents, which aligns well with prior research. Respondents especially note the value the Internet provides in affording connection with other individuals with bipolar disorder, who can relate to and understand their own condition with no sense of stigma association. Finally, social media sites provide an opportunity to reminisce using past content, which reminds respondents of the positive moments they have experienced and provides hope and motivation when attempting to pull through periods of depression. 
Prior research focusing on online social interactions (e.g. through email, text messaging, or social networking sites), finds subtleties regarding how technology-mediated connectivity affects well-being and mental health. In particular, the medium of communication matters, as does with whom the communication happens. Studies comparing various types of online and offline sociality find that face-to-face interactions seem more beneficial to mental well-being than online social interactions (Lewandowski, Rosenberg, Parks, \& Siegel, 2011). Online communication with family and friends is found to have positive effects on wellbeing and relieve depressive symptoms, whereas use of the Internet to meet new people and relieve social isolation relates to higher levels of depression (Ahn \& Shin, 2013). In our study, we see similar tensions related to whether social aspects of technology have positive or negative impacts on the mood of individuals with bipolar disorder. More specifically, we found that technology enables seeking and receiving of social support while also introducing perceptions of paranoia and social anxiety.

\section{Limitations \& future work}

The goal of this study was to assess the perceptions of people with bipolar disorder about their technology use. Our recruitment method (i.e. online survey) hence targeted individuals who had a priori access to technology. Further, participants were self-selecting and their selfreported diagnosis was not externally verifiable. Consequently, our results have limited generalizability to the broader BD population and additional work is needed to provide more evidence to support our findings.

Future research should further investigate the potential of technology-mediated behavior to detect and predict changes in the mental health of people with bipolar disorder. Such work should seek to collect objective data on technology use (e.g., email records, social media data) from people with bipolar disorder. This should also include both further understanding qualitatively the way some individuals use technology to improve their health and their practices around limiting the negative impact of technology. In addition, it would be worthwhile to identify whether there are noticeable differences when it comes to technology use between individuals with different forms of BD (e.g., those who experience psychosis and those who do not).

Beyond technology use serving as a warning sign, there is promise for technology-based protective approaches that diminish the negative impact of technology use for these individuals. This could include protecting against destructive spending sprees that can affect people for many years afterward, artificially diminishing the performance of the person's device to prevent or slow down interactions (e.g., slower to open tabs, running searches etc.) so as not facilitate behavior which exacerbates a person's condition or which they may find overstimulating, providing support and protection to stabilize biological functioning (e.g. filtering circadian affecting light from devices etc.). Given the impairment on decision making particularly present during manic periods this should encompass advanced directive approach currently used for other interventions, where an individual provides legal consent for an intervention while in a healthy state. 


\section{Conclusion}

This paper has explored, from the perspectives of individuals with bipolar disorder, the ways behavior is expressed and shaped via technology. Our findings suggest that technology acts as a double-edged sword for people with bipolar, in that it can both worsen symptoms of the condition as well as enable recovery toward emotional stability. Technology is not one thing for these individuals but variously a source of morale support, stimulation, or anxiety, amongst many others. In summary, this work has helped us gain a broad understanding of the use and ownership of technology by individuals with bipolar disorder. It also indicates that technology use in general may serve as a proactive warning sign of relapse and that content-free privacyprotective features such as use frequency and timing may be effective alone. This knowledge will be relevant to researchers developing behavioral and technological interventions to support bipolar management. There are opportunities for novel interventions to offset some of the negative aspects of technology and to harness its existing strengths.

\section{Acknowledgements}

Removed for anonymous review.

\section{Funding source}

E.L.M. is supported by the National Science Foundation Graduate Research Fellowship under Grant No. DGE 1144153. M.M. work was supported by a Marie Curie Fellowship (Project Number: $\underline{302530}$ ).

\section{References}

$1 \mathrm{D}$. Ahn, D.-H. Shin Is the social use of media for seeking connectedness or for avoiding social isolation? Mechanisms underlying media use and subjective well-being Computers in Human Behavior, 29 (2013), pp. 2453-2462

2 J. Alvarez-Lozano, V. Osmani, O. Mayora, M. Frost, J. Bardram, M. Faurholt-Jepsen, et al. Tell me your apps and I will tell you your mood: correlation of apps usage with bipolar disorder state Proceedings of the 7th International Conference on PErvasive Technologies Related to Assistive Environments, ACM (2014, May), p. 19

3 A. American Psychiatric Diagnostic and statistical manual of mental disorders (DSM-5®) American Psychiatric Pub (2013)

4 R.J. Baldessarini, L. Tondo Suicide risk and treatments for patients with bipolar disorder JAMA, 290 (2003), pp. 1517-1519

5 D. Ben-Zeev, K.E. Davis, S. Kaiser, I. Krzsos, R.E. Drake Mobile technologies among people with serious mental illness: Opportunities for future services Administration and Policy in Mental Health and Mental Health Services Research, 40 (2013), pp. 340-343 
6 K. Bessiere, S. Kiesler, R. Kraut, B.S. Boneva Effects of Internet use and social resources on changes in depression Information, Community \& Society, 11 (2008), pp. 47-70

7 J.M. Blum, E. Magill The design and evaluation of personalised ambient mental health monitors Consumer communications and networking conference (CCNC), 2010 7th IEEE, IEEE (2010), pp. 1-5

8 M.L. Bruce, R.A. Hoff Social and physical health risk factors for first-onset major depressive disorder in a community sample Social Psychiatry and Psychiatric Epidemiology, 29 (1994), pp. 165-171

9 S.E. Caplan Preference for online social interaction a theory of problematic Internet use and psychosocial well-being Communication Research, 30 (2003), pp. 625-648

10 E. Dahlstrom, J.D. Walker, C. Dziuban ECAR study of undergraduate students and information technology (2013)

11 M. De Choudhury, M. Gamon, S. Counts, E. HorvitzPredicting depression via social media (2013)

12 M. Faurholt-Jepsen, M. Frost, C. Ritz, E.M. Christensen, A.S. Jacoby, R.L. Mikkelsen, et al. Daily electronic self-monitoring in bipolar disorder using smartphones-the MONARCA I trial: A randomized, placebo-controlled, single-blind, parallel group trial Psychological Medicine, 45 (2015), pp. 2691-2704

13 J. Firth, J. Cotter, J. Torous, S. Bucci, J.A. Firth, A.R. Yung Mobile phone ownership and endorsement of "mHealth" among people with psychosis: A meta-analysis of cross-sectional studies Schizophrenia Bulletin (2015) sbv132

14 E. Frank, H.A. Swartz, E. Boland Interpersonal and social rhythm therapy: An intervention addressing rhythm dysregulation in bipolar disorder Dialogues in Clinical Neuroscience, 9 (2007), p. 325

15 M. Frost, A. Doryab, M. Faurholt-Jepsen, L.V. Kessing, J.E. Bardram Supporting disease insight through data analysis: Refinements of the monarca self-assessment system Proceedings of the 2013 ACM international joint conference on Pervasive and ubiquitous computing, ACM (2013), pp. 133-142

16 A. Grünerbl, A. Muaremi, V. Osmani, G. Bahle, S. Oehler, G. Tröster, ..., P. Lukowicz Smartphone-based recognition of states and state changes in bipolar disorder patients IEEE Journal of Biomedical and Health Informatics, 19 (1) (2015), pp. 140-148

17 E.C. Harris, B. Barraclough Suicide as an outcome for mental disorders. A metaanalysis The British Journal of Psychiatry, 170 (1997), pp. 205-228

18 A. Harvey Sleep and circadian rhythms in bipolar disorder: Seeking synchrony, harmony, and regulation American Journal of Psychiatry, 165 (2008), pp. 820-829

19 J. Harwood, J.J. Dooley, A.J. Scott, R. Joiner Constantly connected-The effects of smart-devices on mental health Computers in Human Behavior, 34 (2014), pp. 267272

20 L.L. Judd, H.S. Akiskal, P.J. Schettler, W. Coryell, J. Endicott, J.D. Maser, et al. A prospective investigation of the natural history of the long-term weekly symptomatic status of bipolar II disorder Archives of General Psychiatry, 60 (2003), pp. 261-269 
$21 \mathrm{D}$. Kardefelt-Winther A conceptual and methodological critique of internet addiction research: Towards a model of compensatory internet use Computers in Human Behavior, 31 (2014), pp. 351-354

22 E. Kross, P. Verduyn, E. Demiralp, J. Park, D.S. Lee, N. Lin, et al. Facebook use predicts declines in subjective well-being in young adults PloS One, 8 (2013), p. e69841

23 S. Lal, J. Dell'Elce, A.K. Malla Technology access and use among young adults with a first episode of psychosis Psychiatric Services, 66 (7) (2015), pp. 764-765

24 S.D. Lambert, C.G. Loiselle Health information-seeking behaviorQualitative Health Research, 17 (2007), pp. 1006-1019

25 J. Lewandowski, B.D. Rosenberg, M.J. Parks, J.T. Siegel The effect of informal social support: Face-to-face versus computer-mediated communication Computers in Human Behavior, 27 (2011), pp. 1806-1814

26 J. Lofland, L.H. Lofland Analyzing social settings Wadsworth Publishing Company, San Francisco (1995)

27 K. Magsamen-Conrad, K. Greene Technology addiction's contribution to mental wellbeing: The positive effect of online social capital Computers in Human Behavior, 40 (2014), pp. 23-30

28 T. Martini, L.S. Czepielewski, A. Fijtman, L. Sodré, B. Wollenhaupt-Aguiar, C.S. Pereira, et al. Bipolar disorder affects behavior and social skills on the internet PloS One, 8 (2013), p. e79673

29 M. Matthews, S. Abdullah, E. Murnane, S. Voida, T. Choudhury, G. Gay, et al. Development and evaluation of a smartphone-based measure of social rhythms for bipolar disorder Assessment (2016), pp. 1073-1911 1073191116656794

30 K.R. Merikangas, H.S. Akiskal, J. Angst, P.E. Greenberg, R. Hirschfeld, M. Petukhova, et al. Lifetime and 12-month prevalence of bipolar spectrum disorder in the National Comorbidity Survey replication Archives of General Psychiatry, 64 (2007), p. 543

31 K.R. Merikangas, R. Jin, J.P. He, R.C. Kessler, S. Lee, N.A. Sampson, ..., M. Ladea

Prevalence and correlates of bipolar spectrum disorder in the world mental health survey initiative Archives of General Psychiatry, 68 (3) (2011), pp. 241-251

32 D.J. Miklowitz, L.B. Alloy Psychosocial factors in the course and treatment of bipolar disorder: Introduction to the special section Journal of Abnormal Psychology, 108 (1999), p. 555

33 T. Millon, C. Millon, R.D. Davis, S. Grossman Millon clinical multiaxial inventory-III (MCMI-III): Manual Pearson/PsychCorp (2009)

34 C.J. Murray, A.D. Lopez The global burden of disease, Vol. 1, Harvard University Press, Cambridge, MA (1996)

35 L.D. Muusses, C. Finkenauer, P. Kerkhof, C.J. Billedo A longitudinal study of the association between compulsive internet use and wellbeing Computers in Human Behavior, 36 (2014), pp. 21-28

36 R. Nusslock, L. Abramson, E. Harmon-Jones, L. Alloy, J. Coan Psychosocial interventions for bipolar disorder: Perspective from the behavioral approach system (BAS) dysregulation theory Clinical Psychology: Science and Practice, 16 (2009), pp. 449-469 
37 A. Pari, A. Ahmed, J. Simon, J. Wolstenholme, J.R. Geddes, G.M. Goodwin Economic evaluations in bipolar disorder: A systematic review and critical appraisal Bipolar Disorders, 16 (2014), pp. 557-582

38 S. Park, K.-E.M. Hong, E.J. Park, K.S. Ha, H.J. Yoo The association between problematic internet use and depression, suicidal ideation and bipolar disorder symptoms in Korean adolescents Australian and New Zealand Journal of Psychiatry, 47 (2013), pp. 153-159

39 J. Proudfoot, J. Doran, V. Manicavasagar, G. Parker The precipitants of manic/hypomanic episodes in the context of bipolar disorder: A review Journal of Affective Disorders, 133 (2011), pp. 381-387

40 L.D. Rosen, K. Whaling, S. Rab, L.M. Carrier, N.A. Cheever Is Facebook creating "iDisorders"? The link between clinical symptoms of psychiatric disorders and technology use, attitudes and anxiety Computers in Human Behavior, 29 (2013), pp. 1243-1254

41 A. Smith The smartphone difference Pew Research Center (2015)

42 A.A. Stone, S. Shiffman, J.E. Schwartz, J.E. Broderick, M.R. HuffordPatient compliance with paper and electronic diaries Controlled Clinical Trials, 24 (2003), pp. 182-199

43 T. Suppes, G.S. Leverich, P.E. Keck, W.A. Nolen, K.D. Denicoff, L.L. Altshuler, et al. The Stanley Foundation bipolar treatment outcome network: II. Demographics and illness characteristics of the first $\mathbf{2 6 1}$ patients Journal of Affective Disorders, 67 (2001), pp. 45-59

44 S. Thomée, A. Härenstam, M. Hagberg Mobile phone use and stress, sleep disturbances, and symptoms of depression among young adults-a prospective cohort study BMC Public Health, 11 (2011), p. 1

45 G. Valenza, M. Nardelli, A. Lanata, C. Gentili, G. Bertschy, R. Paradiso, et al. Wearable monitoring for mood recognition in bipolar disorder based on history-dependent long-term heart rate variability analysis IEEE Journal of Biomedical and Health Informatics, 18 (2014), pp. 1625-1635

46 B. Wood, M.S. Rea, B. Plitnick, M.G. Figueiro Light level and duration of exposure determine the impact of self-luminous tablets on melatonin suppression Applied Ergonomics, 44 (2013), pp. 237-240

47 K.S. Young Internet addiction: The emergence of a new clinical disorder CyberPsychology \& Behavior, 1 (1998), pp. 237-244

Appendix A. Supplementary data

The following is the supplementary data related to this article: 


\section{APPENDIX}

Please indicate how much you agree or disagree with each of the following statements.

\begin{tabular}{|c|c|c|c|c|c|}
\hline & Strongly Agree & Agree & $\begin{array}{l}\text { Neither Agree } \\
\text { nor Disagree }\end{array}$ & Disagree & $\begin{array}{l}\text { Strongly } \\
\text { Disagree }\end{array}$ \\
\hline $\begin{array}{c}\text { I'm } \\
\text { enthusiastic } \\
\text { about } \\
\text { electronics } \\
\text { and digital } \\
\text { devices }\end{array}$ & O & O & $\mathrm{O}$ & $\mathrm{O}$ & 0 \\
\hline $\begin{array}{l}\text { I frequently } \\
\text { look for new } \\
\text { software or } \\
\text { apps }\end{array}$ & O & 0 & 0 & O & $\bigcirc$ \\
\hline $\begin{array}{l}\text { My friends } \\
\text { would } \\
\text { describe me } \\
\text { as "into" the } \\
\text { latest } \\
\text { technology }\end{array}$ & O & 0 & 0 & 0 & 0 \\
\hline $\begin{array}{l}\text { For me, } \\
\text { technology is } \\
\text { frustrating }\end{array}$ & O & O & $\mathrm{O}$ & $\mathrm{O}$ & O \\
\hline
\end{tabular}

